\title{
Direct Drive Position Control of a Spiral Motor as a Monoarticular Actuator
}

\author{
Ahmad Zaki Shukor, Member, IEEE and Yasutaka Fujimoto, Senior Member, IEEE
}

\begin{abstract}
This paper presents the direct drive position control verification of a spiral motor in monoarticular configuration. The spiral motor is a newly developed high thrust, high backdrivable direct drive three-phase PM motor with a unique 3D structure. One of the possible uses of the spiral motor is to actuate musculoskeletal-like structure which is shown in animals and humans. This is achieved by indirectly actuating the elbow/shoulder joints via pulling/pushing the links by using a linear actuator. We describe the control methods for the direct drive of the spiral motor which are decoupling and independent control. Next simulations were performed to assess control parameter variation effects. Then the experimental results confirm the validity of the control method in monoarticular position. The key element of linear motion control of the spiral motor is the magnetic levitation (air gap between stator and rotor) control and simultaneous angular motion control via vector $d$ - and $q$-axis currents.
\end{abstract}

Index Terms-spiral motor, musculoskeletal actuation, direct drive, PM motor, vector control.

\section{NOMENCLATURE}

$\begin{array}{ll}I_{d}, I_{q} & \text { d-axis and q-axis current. } \\ \theta & \text { Mechanical rotation angle of the rotor. } \\ x & \text { Linear displacement of rotor. } \\ x_{g} & \text { Air gap displacement. } \\ K_{f} & \text { Thrust force constant. } \\ K_{\tau} & \text { Torque constant. } \\ K_{g} & \text { Magnetic levitation constant. } \\ d_{x}, d_{\theta} & \text { Thrust force and torque disturbances including } \\ & \text { modeling error. } \\ d_{x g} & \text { Gap disturbance. } \\ l_{p} & \text { Lead length of screw. } \\ u_{\theta} & \text { Rotational control variable. } \\ u_{x g} & \text { Gap control variable. } \\ M_{s} & \text { Mass of the rotor. } \\ J_{s} & \text { Moment of inertia of the rotor. } \\ g_{x} & \text { Thrust disturbance observer gain. } \\ g_{\theta} & \text { Torque disturbance observer gain. } \\ g_{x g} & \text { Gap disturbance observer gain. } \\ \omega_{1} & \text { Cutoff frequency for rotational motion. } \\ \omega_{2} & \text { Cutoff frequency for linear motion. }\end{array}$

Manuscript received October 13, 2012. Accepted for publication April 22, 2013.

Copyright 2009 IEEE. Personal use of this material is permitted. However, permission to use this material for any other purposes must be obtained from the IEEE by sending a request to pubs-permissions@ieee.org.

A. Z. Shukor was with the Department of Electrical and Computer Engineering, Yokohama National University, Yokohama 240-8501 Japan. He is now with the Faculty of Electrical Engineering, Universiti Teknikal Malaysia Melaka (e-mail: zaki@ fujilab.dnj.ynu.ac.jp).

Y. Fujimoto is with the Department of Electrical and Computer Engineering, Yokohama National University, Yokohama 240-8501 Japan (e-mail: fujimoto@ynu.ac.jp).

$\begin{array}{ll}x_{g}^{c m d} & \text { Gap position command reference. } \\ K_{p g} & \text { Proportional control gain for air gap. } \\ K_{d g} & \text { Derivative control gain for air gap. } \\ \theta^{c m d} & \text { Angle position command reference. } \\ K_{p \tau} & \text { Proportional control gain for angle. } \\ K_{d \tau} & \text { Derivative control gain for angle. } \\ K_{p i} & \text { Proportional gain for current regulator. } \\ K_{i} & \text { Integral gain for current regulator. }\end{array}$

\section{INTRODUCTION}

$\mathbf{R}$ OBOTICS have shown rapid developments in the past decades. We have grown accustomed to industrial robots that assist us in production lines in manufacturing such as KUKA [1], FANUC [2] and many others. We have also assimilated mobile robots in our everyday lives/work to perform various tasks such as material handling or inspection in hazardous environments, space exploration as well as entertainment or services that involve interaction with humans or objects.

One of the reasons for successful integration of robots in our living/working space is the actuator that enables motion. It is considered one of the most important outputs of a robot to perform tasks. Actuators could involve hydraulics, pneumatics, electrical motors or other active or passive mechanisms. An example of a mobile robot that mimics musculoskeletal actuation using hydraulics and electrical motors is the HyQ four-legged robot [3]. It comprises of 12 active degrees of freedom (dof) that uses brushless DC motors with harmonic gears for hip abduction/adduction for lateral leg motion and hydraulic cylinders with high performance servo vales (250 $\mathrm{Hz}$ bandwith) for knee flexion/extension. Impedance control was used for compliant actuation. Another example of a fourlegged full hydraulic-actuated robot is Bigdog [4] which is under development at Boston Dynamics with funding from DARPA. Researchers claim the robot can carry $154 \mathrm{~kg}$ on flat terrain. The robot shows very stable walking control even on uneven or slippery terrain or downhill walk.

For pneumatics-actuated designs, researchers in [5] utilized pneumatic artificial muscles (PAM) to construct a legged robot that can realize compliance control. Position tracking control for frequencies up to $3 \mathrm{~Hz}$ were tested and collision experiments for were also performed to demonstrate the compliance of the legged structure using methods which are independent joint compliance control and sliding mode control. After collision, low forces were shown for both methods thus showing enhanced safety using pneumatic muscles. Other examples of pneumatic-actuated robots are seen in [6] and [7]. In [6], the monopod can perform jumping motion and in [7], the robot that resembles a frog structure can perform jumping motions. 
On the other hand, elastically-actuated robots suggests another option for safety (i.e working alongside humans). A biped robot utilizing servo motors with elastomers on the load side placed at each joints was shown in [8]. It can be categorized as a series-elastic-actuator robot. Position and force control were realized using disturbance observers at the elastomer side and the output side. Tracking for position and force were constructed using high-order derivatives and could be an option for compliance control. Another example of series elastic actuated robot is the cCub robot [9]. The robot uses a new design of compliant actuator (CompAct) which has high torsion stiffness and at the same time, large passive deflection ranges, in other words variable physical damping actuators [10]. The validity of the actuator in replicating desired values of joint viscous damping was shown. A rotational series elastic actuator (RSEA) for use in human assistive systems were reported in [11]. The design uses a $150 \mathrm{~W}$ Maxon DC motor, worm gear, worm wheel, torsional spring and spur gear to experimentally realise the power assist of human leg.

Electrical motors are also used for redundantly actuated musculoskeletal-inspired designs, such as in [12] and [13]. In [12], redundant biarticular (two-joint) actuation were realized by motors and wires for the complete six muscles from the shoulder joint to the elbow joint. The motor pulls or releases the wires to mimic antagonistic motion of the human arm muscles. For the Lancelet robot in [13], sigmoid swimming motion were implemented using virtual triarticular (threejoint) actuation. Motors and pulleys were used to actuate the robot. For a biarticular manipulator, its hexagonal force ellipsoid is an advantage compared to monoarticular actuation. Force maximization using infinity norm for the BiarticularWire-driven robot was experimentally verified and compared with two-norm approach in [14]. A biarticular structure using DC motors and planetary gears was also shown in [15]. Other researchers use electromagnetic linear actuators for their biped design, such as in [16] and [17]. The actuator in [16] is a direct drive 3-phase synchronous motor which is able to generate thrust of $5.7 \mathrm{~N}$ with an effective current of $1 \mathrm{~A}$. For [17], linear induction motors with neodymium magnets were used for their biped robot. The shortest stroke of the motor is 50 $\mathrm{mm}$ with a force of around $40 \mathrm{~N}$.

A good option for direct drive linear actuator is the tubular linear permanent magnet synchronous motor (TLPMSM). In general, this type of motor is a very efficient direct drive linear actuator because of the tubular design of rotor integrated with permanent magnets and stator windings generates enough current to magnetically levitate the rotors and perform direct drive. Many designs of TLPMSM are researched over the years. In [18], the TLPMSM replaces the use of rotational electrical induction motor for refrigerator compressors. Other than reduction of frictional loss, the actuator increases the efficiency by modulating the refrigerator load according to demand. Another application of TLPMSM is for active suspension control in [19]. The quarter-car test bed was constructed using the motor and modified lead-lag, LQ servo and fuzzy controllers were implemented to attenuate road disturbance. Fuzzy controllers turned out to be the most suitable control due to its performance. A recent interesting design of TLPMSM is the Bendable Permanent Magnet Tubular Linear Motor [20]. It is a flexible two-phase linear actuator which uses elastomer rings between the coils of the stator and between magnets in the rotor. Experiments were performed and compared between bent and unbent modes with minimum and maximum strokes. A cryogenic tubular linear actuator design and experiments were implemented with Liquid Nitrogen cooling to minimize the power losses were reported in [21]. A tubular transverse flux machine with permanent magnet excitation was developed and analysed in [22]. With regards to magnetic levitation, several control methods were investigated by other researchers. For example, Adaptive Sliding Mode Controller was used for a Macro-Micro Planar Maglev positioning system in [23]. PID and Particle Swarm Optimization (PSO) were used in another Maglev transportation system using Linear Induction Motor in [24]. A research on high frequency voltage injection on Linear Permanent Magnet Synchronous Motor (LPMSM) using compensated position control by exploiting d-axis high frequency current component was shown in [25].

A common linear motor can be divided into direct-drive and non direct-drive motors. Direct-drive linear motors include TLPMSM which is tube-shaped moving element with permanent magnet or LIM (Linear Induction Motors) with flat-shaped moving element. Example of non direct-drive linear motors is a rotating motor (i.e servo) with a coupled mechanism such as ball screw [26] or rack and pinion gears or pulleys or belts to realize linear motion. The advantages of a spiral motor compared to common linear motor are the high thrust density per volume output and realization of directdrive using magnetic levitation between helical-shaped stator and rotor. The first spiral motor prototype was a large thrust force actuator (around $2000 \mathrm{~N}$ ) that was shown in [27]. Then, the compact size surface permanent magnet spiral motor was reported in [28]. The size is a multifold reduction than the first prototype because the current prototype is intended for musculoskeletal actuation. Simulation of workspace control of the generalized closed-kinematic chain biarticular structure was shown in [29] and force control and load effects using spiral motors shown in [30] [31].

This paper is organized as follows, Section II introduces the spiral motor structure and simplified model, Section III explains the direct drive control methods for the spiral motor, Section IV shows the simulation results for direct drive control methods and Section $\mathrm{V}$ presents the direct drive experimental results for the spiral motor.

\section{THE SPIRAL MOTOR}

The spiral motor is a helically-shaped three-phase permanent magnet synchronous linear motor. It is a compact, high forward and backward drivable and high thrust permanent magnet motor because flux is effectively utilized in threedimensional structure. The stator yoke is made of soft magnetic compost and the helical-shaped permanent magnet is made of Nd-Fe-B. The internal (illustrated) structure of the motor can be seen in Fig. 1 and the exterior (assembled) structure can be seen in Fig. 2. The stator block and rotor unit are shown in Fig. 3 and half-assembled stator and rotor 
are shown in Fig. 4. By using rotary and linear encoder, the internal air gap $x_{g}$ between the stator and rotor can be measured via the following equation:

$$
x_{g}=x-\frac{l_{p}}{2 \pi} \theta
$$

Linear encoder measures linear displacement $x$ and rotary encoder measures rotational displacement $\theta$. The lead length of screw $l_{p}$ is $20 \mathrm{~mm}$ which means that if the gap is maintained at $0 \mathrm{~mm}$ (centre) position, one revolution of rotational displacement $\theta$ will result in a linear displacement of $20 \mathrm{~mm}$. The magnet is attached to teflon sheet, thus the ideal distance (gap) between the teflon sheet and stator yoke is $700 \mu \mathrm{m}$ in both directions of thrust. However, due to manufacturing accuracies, gap lengths vary for each rotation of angle.

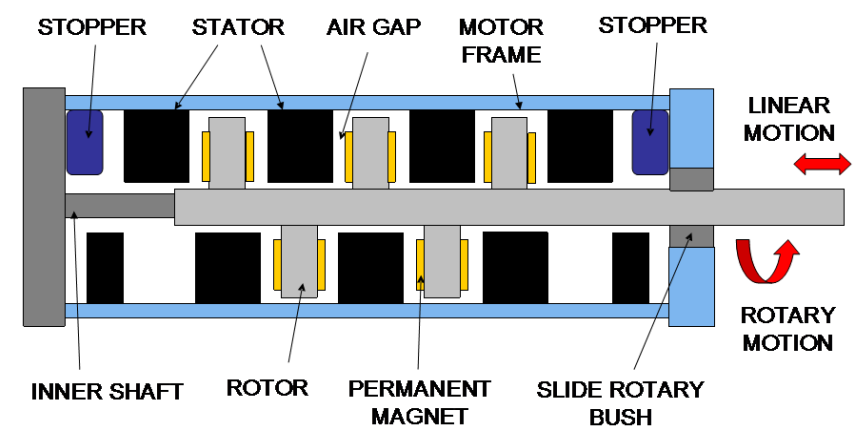

Fig. 1. Internal (illustrated) view of spiral motor

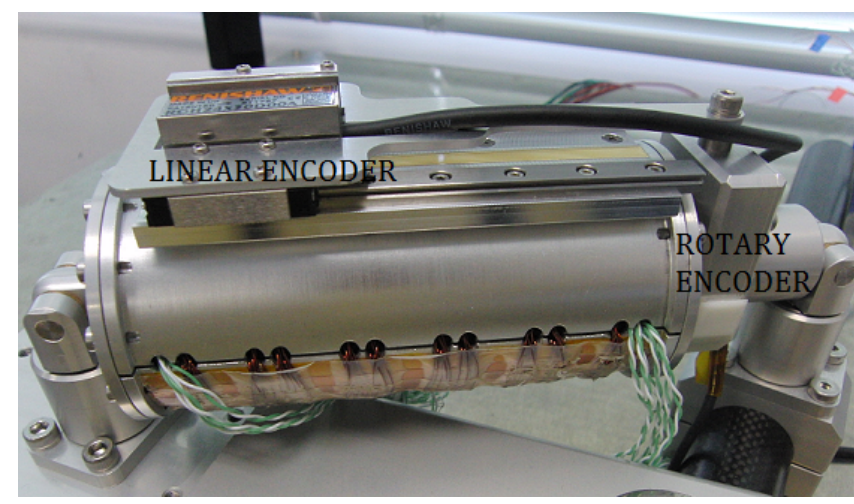

Fig. 2. Exterior (assembled) short-stroke spiral motor

The detailed magnetic circuit permeance model of the motor can be further read in [28]. However, we intend to use the simplified model of the spiral motor which is described by the following equations:

$$
\begin{aligned}
M_{s} \ddot{x} & =K_{f} I_{d}+K_{g} x_{g}-d_{x} \\
J_{s} \ddot{\theta} & =K_{\tau} I_{q}-h\left(K_{f} I_{d}+K_{g} x_{g}\right)-d_{\theta} \\
h & =\frac{l_{p}}{2 \pi}
\end{aligned}
$$

The thrust force of the spiral motor is shown as $M_{s} \ddot{x}$, a product of the mass with linear acceleration while the rotational torque of the motor is shown as $J_{s} \ddot{\theta}$, inertia multiplied with rotational acceleration. Thus the motor can be described as a 2 dof plant. $K_{f} I_{d}$ is the force from d-axis current and $K_{g} x_{g}$ is magnetic levitation force. $d_{x}$ depicts thrust

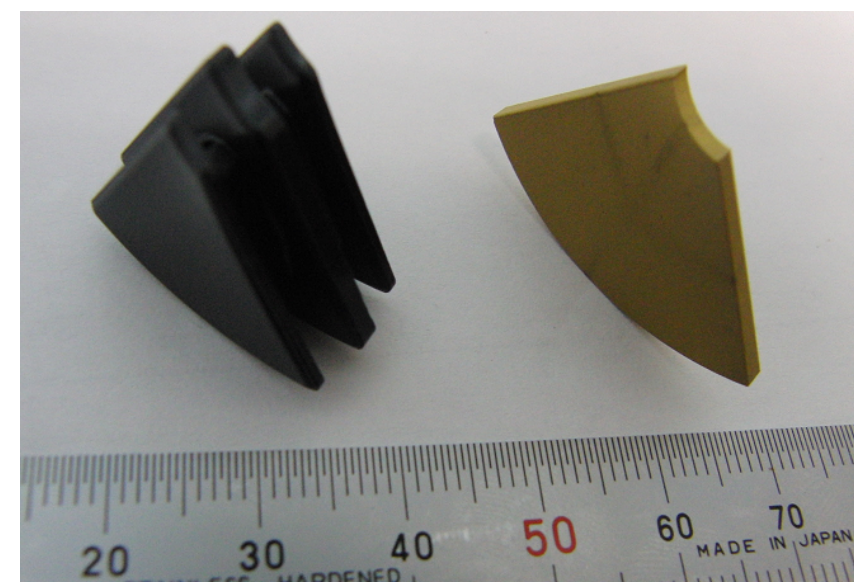

Fig. 3. Small parts: Stator yoke block (left) and rotor magnet (right)

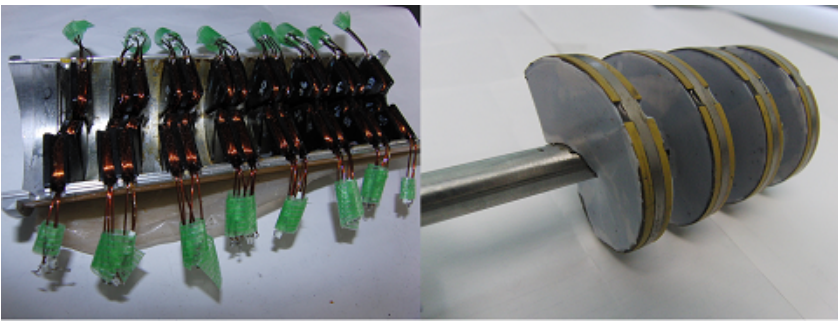

Fig. 4. Half-assembled: Half-stator (left) and rotor unit (right)

disturbance and $d_{\theta}$ is the torque disturbance term. $K_{\tau} I_{q}$ is the force from q-axis current and $-h\left(K_{f} I_{d}+K_{g} x_{g}\right)$ is a coupled term from thrust force. The constants in these equations of motion can be identified via the voltage-current model [28].

The magnetic levitation force term $K_{g} x_{g}$ is a product of gap displacement with gap constant. The larger the value of gap, the larger the magnetic levitation force is. This means that enough currents must be supplied to levitate the rotor to the desired gap position. At center (or neutral) position, forces are zero. However, due to disturbances (i.e manufacturing defects, external disturbance), this neutral point varies.

\section{Direct Drive Control}

\section{A. Decoupling Direct Drive Control}

For the direct drive to be realized, magnetic levitation must be performed. Without magnetic levitation, rotational motion of the rotor will contact the stator and produce large friction forces which is undesired. Thus the key element in direct drive control of our actuator is the magnetic levitation (air gap) control. By rewriting Equations (2) and differentiating (1) twice, the air gap acceleration $\ddot{x}_{g}$ can be seen in (5). The plant equations are written as follows:

$$
\begin{aligned}
M_{s}\left(\ddot{x}_{g}+h \ddot{\theta}\right) & =K_{f} I_{d}+K_{g} x_{g}-d_{x} \\
J_{s} \ddot{\theta} & =K_{\tau} I_{q}-h\left(K_{f} I_{d}+K_{g} x_{g}\right)-d_{\theta}
\end{aligned}
$$

For decoupling direct drive control, assume two control variables $u_{x g}$ depicting gap control term and $u_{\theta}$ as rotational control term. These variables are constructed as follows:

$$
\begin{aligned}
u_{x g} & =K_{p g}\left(x_{g}^{c m d}-x_{g}\right)+K_{d g}\left(\dot{x}_{g}^{c m d}-\dot{x}_{g}\right) \\
u_{\theta} & =\ddot{\theta}^{c m d}+K_{p \tau}\left(\theta^{c m d}-\theta\right)+K_{d \tau}\left(\dot{\theta}^{c m d}-\dot{\theta}\right)
\end{aligned}
$$


The control variables are PD control terms that regulate the errors in each gap or rotational space. The currents $I_{d}$ and $I_{q}$ are virtual inputs to the spiral motor plant. By using vector control strategy, the current references can be generated as the following (subscript $n$ are the controller-assigned values).

$$
\begin{aligned}
I_{\text {dref }} & =\frac{1}{K_{f}}\left(M_{n}\left(u_{x g}+h u_{\theta}\right)-K_{g} x_{g}+\hat{d}_{x}\right) \\
I_{\text {qref }} & =\frac{1}{K_{\tau}}\left(J_{n}\left(u_{\theta}\right)+h\left(M_{n}\left(u_{x g}+h u_{\theta}\right)+\hat{d}_{x}\right)+\hat{d}_{\theta}\right)
\end{aligned}
$$

The coupled terms show that d-axis current affect both gap and rotational variables and q-axis current is also inter-related. Then the disturbance observer terms, $\hat{d}_{x}$ and $\hat{d}_{\theta}$ to estimate the linear and rotational disturbances can be derived.

$$
\begin{aligned}
& \hat{d}_{x}=\frac{g_{x}}{s+g_{x}}\left(K_{f} I_{\text {dref }}+K_{g} x_{g}+M_{n} \hat{\dot{x}}\right)-M_{n} \hat{\dot{x}} \\
& \hat{d}_{\theta}=\frac{g_{\theta}}{s+g_{\theta}}\left(K_{\tau} I_{\text {qref }}-h\left(K_{f} I_{\text {dref }}+K_{g} x_{g}\right)+J_{n} \hat{\dot{\theta}}\right) \\
& \\
& -J_{n} \hat{\dot{\theta}}
\end{aligned}
$$

The linear and rotational velocities are estimated by applying low-pass filters (at cut-off frequencies $\omega_{1}$ and $\omega_{2}$ ) to differentiation of linear and rotational displacements $(\hat{\dot{x}}$ and $\hat{\dot{\theta}}$ ). Also, no further differentiation is needed to obtain accelerations because the disturbance observer design only utilizes velocity terms. Disturbance observer gains are labeled $g_{x}$ and $g_{\theta}$ for linear and angular disturbances. The decoupling controller block diagram is shown in Fig. 5. The blocks ' $\mathrm{X}$ DOB' and 'Theta-DOB' are represented by (11) and (12).

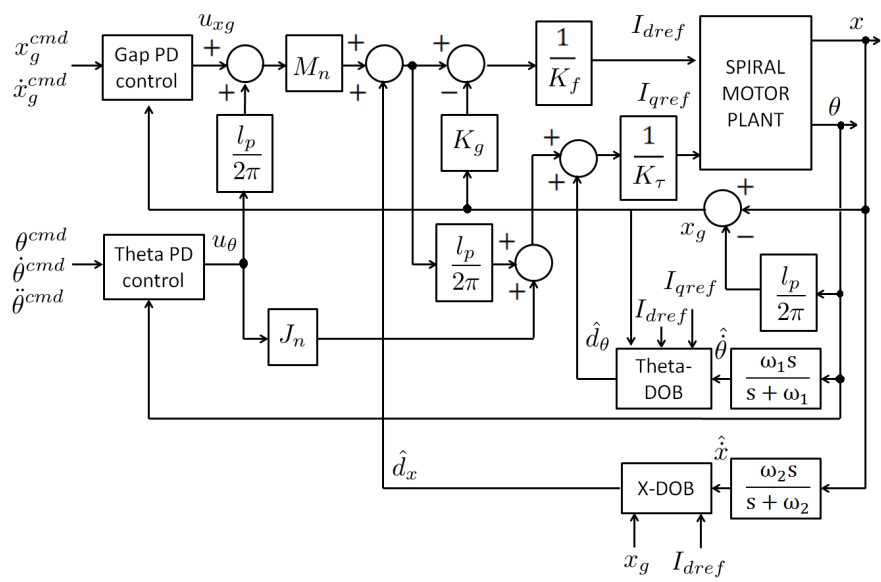

Fig. 5. Decoupling control block diagram for spiral motor

\section{B. Magnetic Levitation using D-axis Current}

For initial verification of gap control, we assign only d-axis current for magnetic levitation. Assuming rotational acceleration terms are zero (no angular motion for rotor), Equation (5) can be estimated as follows:

$$
M_{s}\left(\ddot{x}_{g}\right)=K_{f} I_{d}+K_{g} x_{g}-d_{x g}
$$

Note that disturbance term for linear motion is simplified as gap disturbance $\left(d_{x g}\right)$. Then the required d-axis reference current can be generated as shown in (15).

$$
I_{\text {dref }}=\frac{1}{K_{f}}\left(M_{n}\left(u_{x g}\right)-K_{g} x_{g}+\hat{d}_{x g}\right)
$$

and gap disturbances are estimated as in (15).

$$
\hat{d}_{x g}=\frac{g_{x g}}{s+g_{x g}}\left(K_{f} I_{d r e f}+K_{g} x_{g}+M_{n} \hat{\dot{x}}_{g}\right)-M_{n} \hat{\dot{x}}_{g}
$$

The term $\hat{\dot{x}}_{g}$ represents the gap velocity calculation from estimated linear and rotational velocities.

$$
\hat{\dot{x}}_{g}=\hat{\dot{x}}-h \hat{\dot{\theta}}
$$

\section{Independent Direct Drive Control}

In our preliminary gap control experiments, $d$-axis current controls spiral motor gap with little effect on rotational displacement. Because of this, we attempt to simplify the whole direct drive control of the spiral motor by separating the gap and rotational control terms to each virtual inputs (d-axis and $\mathrm{q}$-axis currents). Using the same control variables for gap and rotational terms, the current references are generated slightly different than decoupling direct drive control, shown in (17) and (18). Disturbances are estimated in (19) and (20).

$$
\begin{gathered}
I_{\text {dref }}=\frac{1}{K_{f}}\left(M_{n}\left(u_{x g}\right)-K_{g} x_{g}+\hat{d}_{x g}\right) \\
I_{\text {qref }}=\frac{1}{K_{\tau}}\left(J_{n}\left(u_{\theta}\right)+\hat{d}_{\theta}\right) \\
\hat{d}_{x g}=\quad \frac{g_{x g}}{s+g_{x g}}\left(K_{f} I_{d r e f}+K_{g} x_{g}+M_{n} \hat{\dot{x}}_{g}\right) \\
\quad-M_{n} \hat{\dot{x}}_{g} \\
\hat{d}_{\theta}=\quad \frac{g_{\theta}}{s+g_{\theta}}\left(K_{\tau} I_{\text {qref }}+J_{n} \hat{\dot{\theta}}\right)-J_{n} \hat{\dot{\theta}}
\end{gathered}
$$

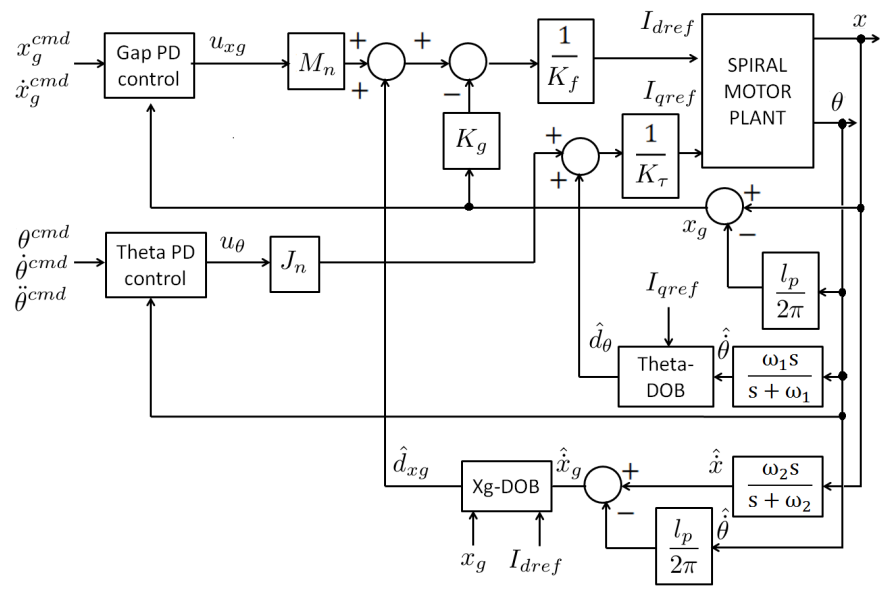

Fig. 6. Independent control block diagram for spiral motor

Note that the disturbance observer for linear motion $\left(g_{x}\right)$ was changed to gap observer $\left(g_{x g}\right)$. Angular disturance is estimated without the coupling term shown in decoupling 


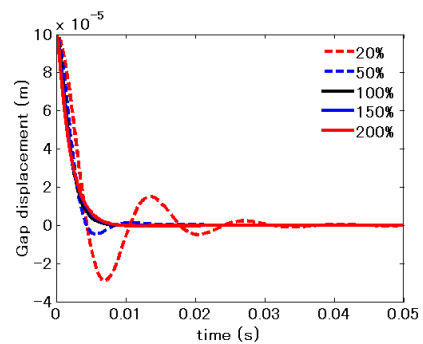

(a)

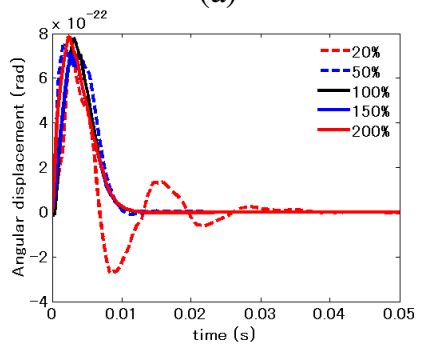

(e)

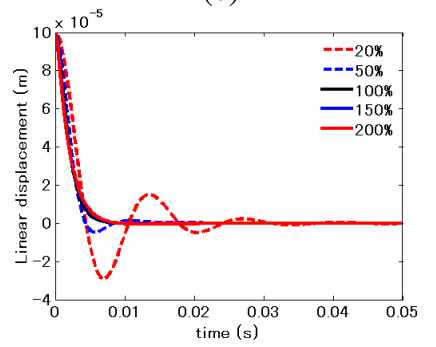

(i)

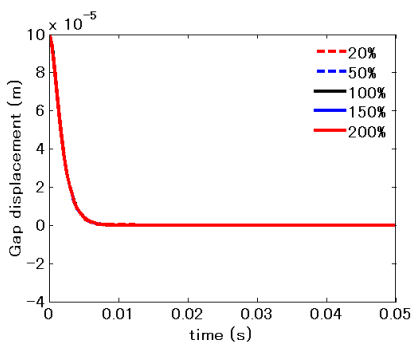

(b)

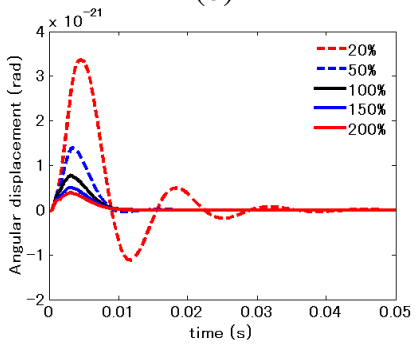

(f)

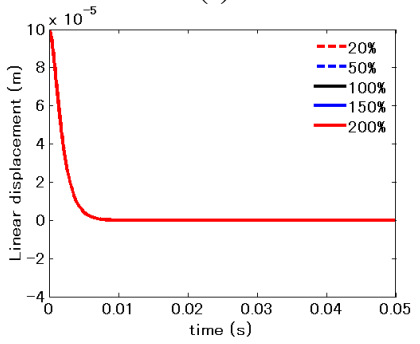

(j)

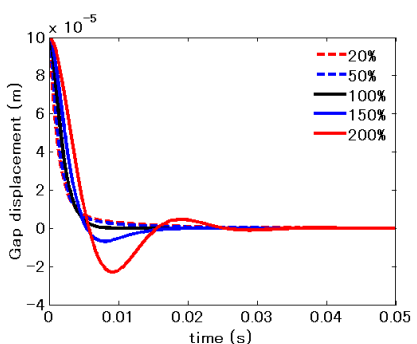

(c)

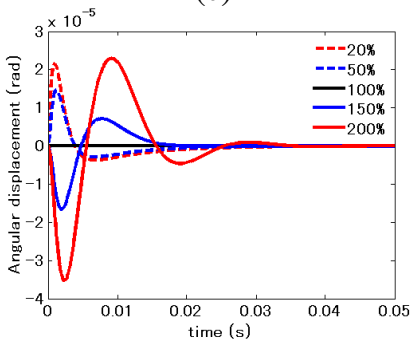

(g)

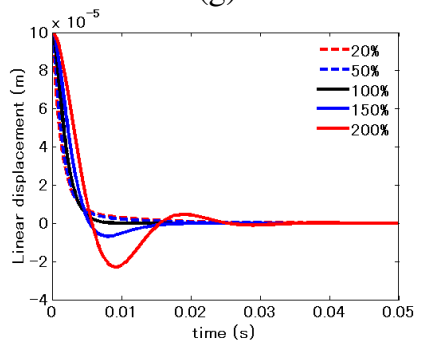

(k)

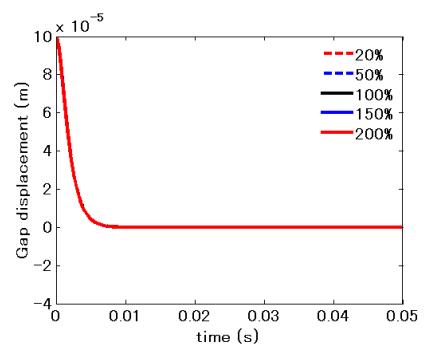

(d)

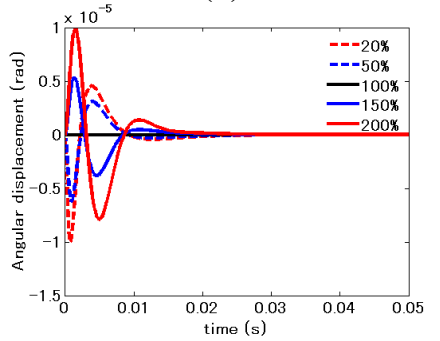

(h)

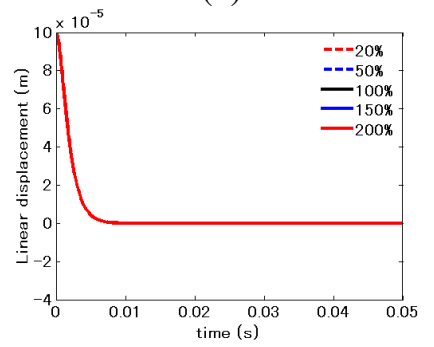

(l)

Fig. 7. Simulation results for decoupling direct drive control; (a)-(d) Gap displacements with varying mass (a), inertia (b), force constant (c) and torque constant(d), (e)-(h) Angular displacements with varying mass (e), inertia (f), force constant (g) and torque constant(h), (i)-(l) Linear displacements with varying mass (i), inertia (j), force constant (k) and torque constant(l)

controller. Also the velocity estimation changes from linear velocity to gap velocity The independent controller block diagram is depicted in Fig. 6. The blocks ' $\mathrm{Xg}$-DOB' and 'Theta-DOB' are represented by (19) and (20).

\section{Simulation of Direct Drive Control}

For initial verification of the two proposed control structure, simulation is performed. The purposes for the variation of parameters are to investigate mass/inertia $\left(M_{n}, J_{n}\right)$ variations that occur when a load/workpiece is attached to the rotor end or link and to predict responses in the case of parameter $\left(M_{n}\right.$, $J_{n}, K_{f}, K_{\tau}$ ) mismatch. For example, mass variations of larger than $100 \%$ represent a certain load placed. The larger the percentage, the larger the load. A low percentage could mean that mass values are not given large enough as responses may deteriorate. The parameters set for the spiral motor plant is described in Table I.

The parameter variations are given at $20 \%, 50 \%, 100 \%$, $150 \%$ and $200 \%$ of the values for $K_{f}, K_{t}, M_{s}$ and $J_{s}$. Control is performed to levitate the magnet from $0.1 \mathrm{~mm}$ (stator-rotor contact position) to $0 \mathrm{~mm}$ and simultaneously maintain angular displacement at $0 \mathrm{rad}$. The responses for gap $x_{g}$, angular displacement $\theta$ and linear displacement $x$ are shown for decoupling direct drive control in Fig. 7. For decoupling control, variations in $M_{s}$ (Fig. 7(a)(e)(i)) affects gap, angle and linear displacement. This is because mass is
TABLE I

SPIRAL Motor PARAMETERS FOR SimUlation

\begin{tabular}{cccc}
\hline \hline Parameters & Symbol & Plant Model Value & Unit \\
\hline Force Constant & $K_{f}$ & 20 & {$[\mathrm{~N} / \mathrm{A}]$} \\
Torque Constant & $K_{\tau}$ & 0.25 & {$[\mathrm{Nm} / \mathrm{A}]$} \\
Gap Constant & $K_{g}$ & 1000000 & {$[\mathrm{~N} / \mathrm{m}]$} \\
Mass & $M_{s}$ & 0.7 & {$[\mathrm{~kg}]$} \\
Inertia & $J_{s}$ & 0.0016 & {$\left[\mathrm{kgm}{ }^{2}\right]$} \\
\hline \hline
\end{tabular}

multiplied with gap and angle control variables. In the case of variations of $J_{s}$ or $K_{\tau}$ (Fig. 7(b)(d)(j)(1)), small variations in angle response occur but gap and linear displacement is least affected. The is due to very small contribution of inertia to gap control variable. If there are variations in force constant $K_{f}$, fluctuations in gap, angle and linear displacement (Fig. $7(\mathrm{c})(\mathrm{g})(\mathrm{k}))$ are observed. Variations in torque constant $K_{\tau}$ only affects angle (Fig. 7(h)). The responses for the independent direct drive are shown in Fig. 8. Although there are some similarities of responses between decoupling control (Fig. 7) and independent control (Fig. 8), larger angle errors occur in independent direct drive Fig. 8(e)-(h). This is because of the coupled term which is not taken into account in the control scheme. However, the errors are small and acceptable. For example, a variation of $1 \times 10^{-5} \mathrm{rad}$ induces $0.03 \mu \mathrm{m}$ linear displacement. 


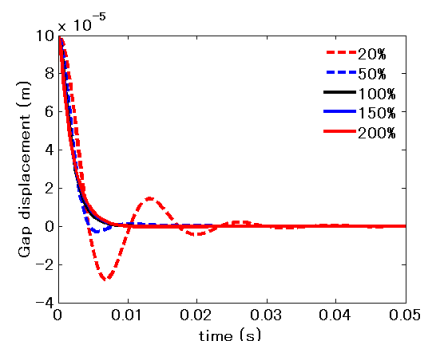

(a)

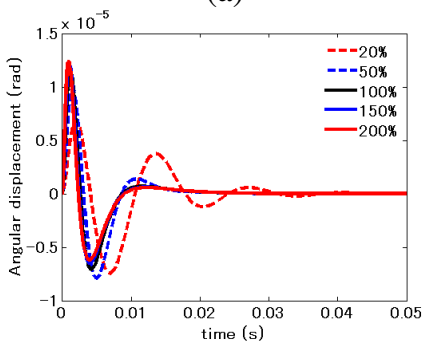

(e)

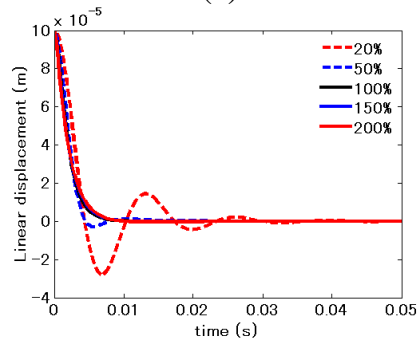

(i)

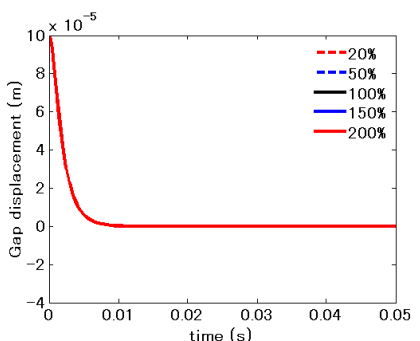

(b)

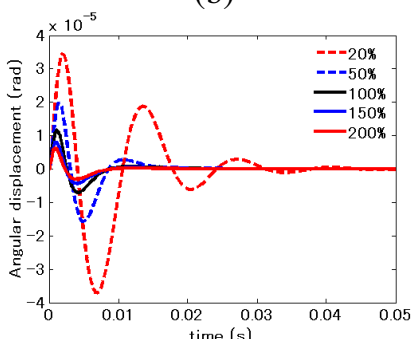

(f)

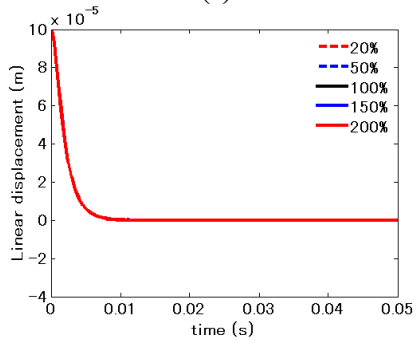

(j)

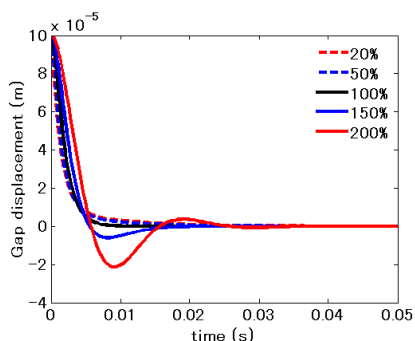

(c)

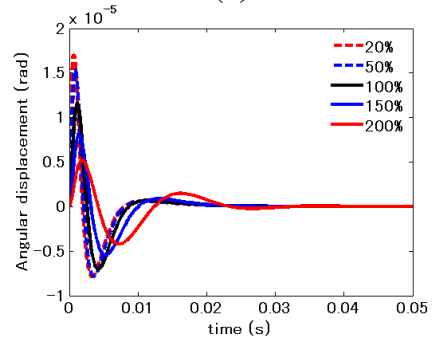

(g)

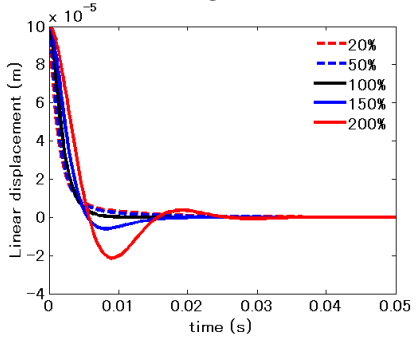

(k)

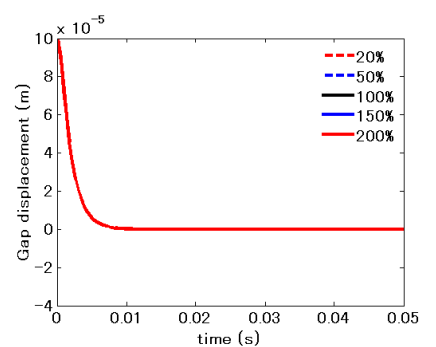

(d)

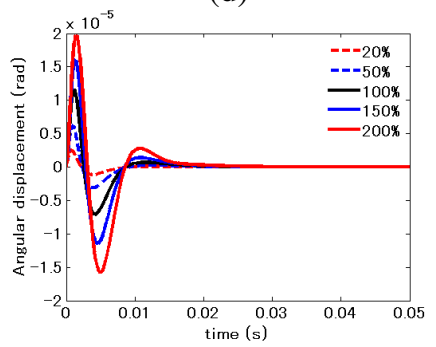

(h)

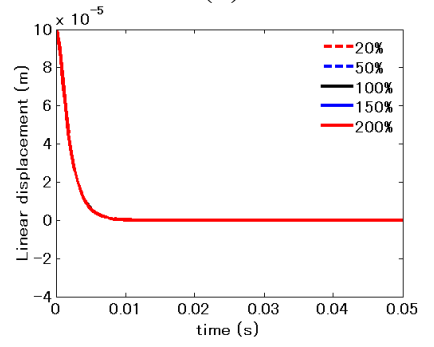

(1)

Fig. 8. Simulation results for independent direct drive control; (a)-(d) Gap displacements with varying mass (a), inertia (b), force constant (c) and torque constant(d), (e)-(h) Angular displacements with varying mass (e), inertia (f), force constant (g) and torque constant(h), (i)-(l) Linear displacements with varying mass (i), inertia (j), force constant $(\mathrm{k})$ and torque constant(1)

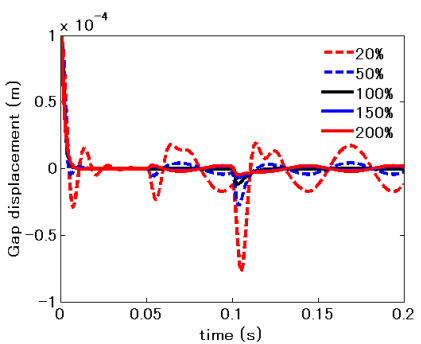

(a)

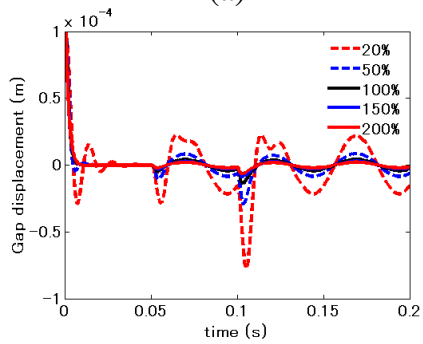

(e)

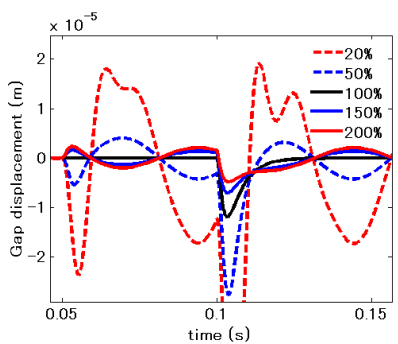

(b)

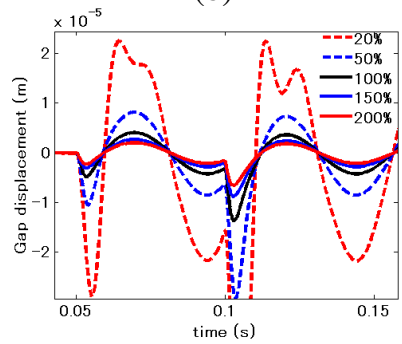

(f)

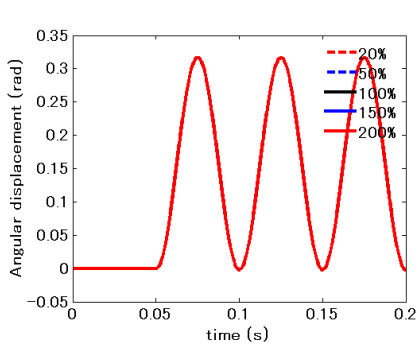

(c)

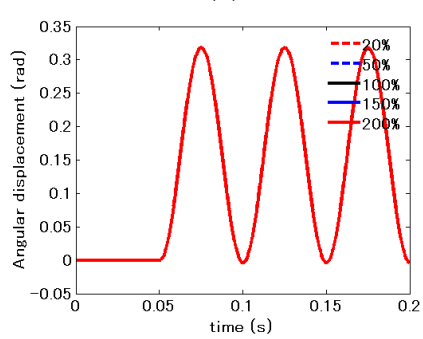

$(\mathrm{g})$

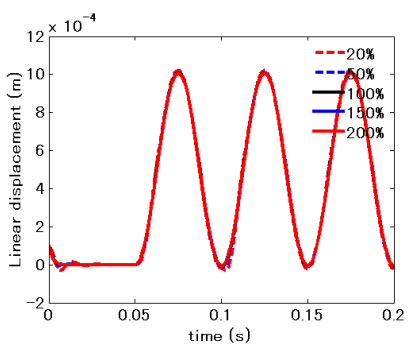

(d)

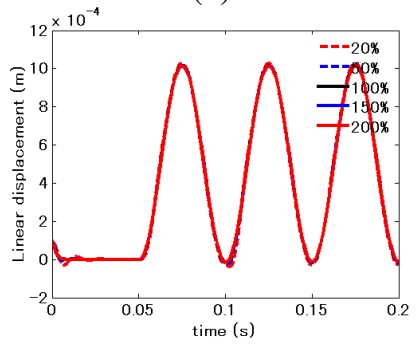

(h)

Fig. 9. Simulation results for sine wave tracking (disturbance starts at $0.1 \mathrm{~s}$ ): decoupling control (a) gap (b) magnified view of gap (c) angle (d), linear displacement, independent control (e) gap (f) magnified view of gap (g) angle (h), linear displacement 
In addition, we include the simulation of $10 \mathrm{~N}$ step linear motion disturbance at $t=0.1 \mathrm{~s}$ in the case of mass $\left(M_{s}\right)$ variations in Fig. 9. Before disturbance injection, linear motion sinusoidal reference was given at $t=0.05 \mathrm{~s}$. Angle and linear displacements are almost similar in both methods. For decoupling control, gap, angle and linear displacements are shown in Fig. 9(a)-(d). After initial stabilization, large gap fluctuations (Fig. 9(a)) for low mass are seen during disturbance injection. At $100 \%$ mass, gap stabilized to zero due to the control scheme that matches the plant model. However, in independent control (Fig. 9(f)), gap errors were not zero throughout simulation. This is expected because the control scheme neglects the coupling term in the plant model. However, these errors are relatively small and shows that even with simplified control, direct drive could be achieved.

\section{DiReCT DRIVE EXPERIMENTS}

For the verification of spiral motor for musculoskeletal actuation, we attach a middle-length $70 \mathrm{~mm}$ stroke spiral motor to the monoarticular configuration, as shown in Fig. 10. This type of structure is a closed-kinematic chain and mimics musculoskeletal actuation for a monoarticular muscle.

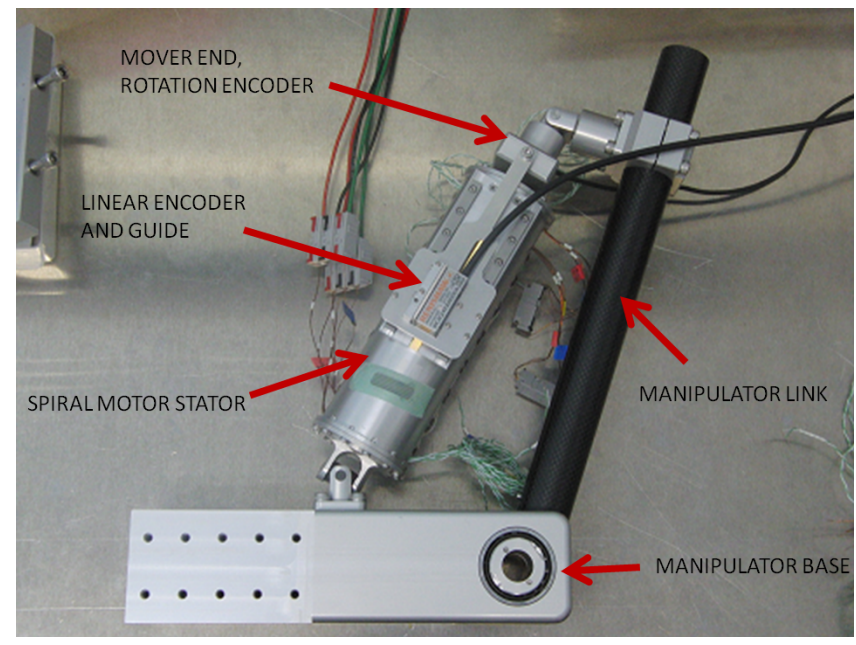

Fig. 10. The spiral motor in monoarticular configuration

For data collection and control, TMS320C6713-225MHz was used. Two three-phase AC PWM inverters for forward and backward drive were supplied by DC voltages. At first, the gaps were given reference to $0 \mathrm{~mm}$ position from an offset of $100 \mu \mathrm{m}$. This means that initially the rotor touches the right side of the stator blocks. The magnetic levitation of spiral motor is illustrated in Fig. 11. Angular displacement is controlled at $0 \mathrm{rad}$ (without any offset). Table II shows the parameters for the experimental system.

Fig. 12 shows the structure of the dq-axis current reference to actual three-phase currents via dq-uvw transformation. Then two DC to AC inverters generates the phase currents. When magnetic levitation is achieved, thrust can be given to the motor by providing angular references for $\mathrm{d}$ - and qaxis currents to track via PI controller. Simultaneously, the magnetic levitation of the rotor must be maintained to avoid friction with stator. Gap variations within these limits are

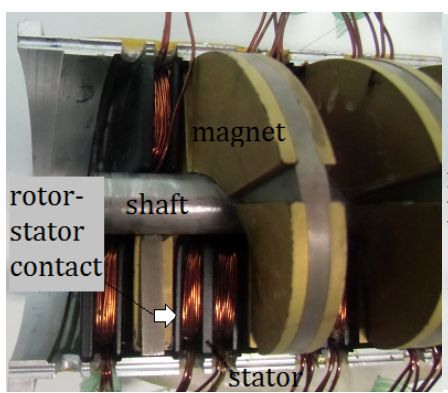

(a)

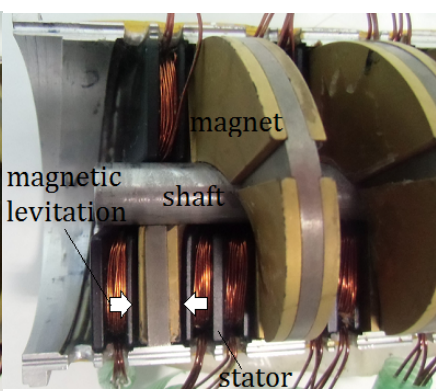

(b)
Fig. 11. Illustration of spiral motor magnetic levitation; motion from touch up position (a) to center $0 \mathrm{~mm}$ position (b)

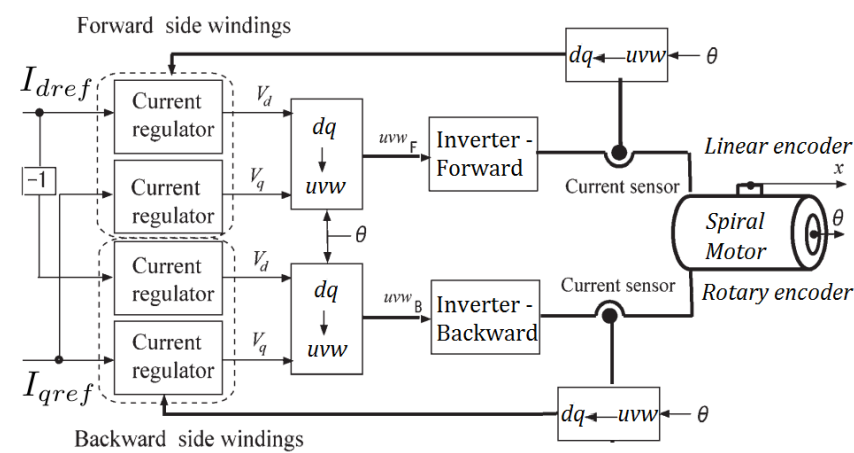

Fig. 12. Illustration of spiral motor inverter structure and coordinate transformation. Current regulator uses PI controller.

acceptable. Initial magnetic levitation from rotor-stator contact (touch-up) position were performed between 0 to $0.5 \mathrm{~s}$. Then from $0.5 \mathrm{~s}$ onwards direct drive control using rotational and gap displacements were carried out. Linear motion reference of $1 \mathrm{~mm}$ is given and the angular reference can be generated as in (21).

$$
\theta_{\text {ref }}=\frac{x_{r e f}-x_{\text {gref }}}{h}=\frac{x_{r e f}-0}{h}
$$

The experimental results are shown in Fig. 13. In the gap responses of both control methods in Fig. 13(a) and (d) (decoupling and independent control), magnetic levitation were achieved from $0.1 \mathrm{~mm}$ to $0 \mathrm{~mm}$ in the gap stabilization phase. Then during angular motion (which produces linear motion), gap were successfully maintained, although more fluctuations existed in the independent control Fig. 13(d) as expected. The position responses in both control Fig. 13(b) and (e) shows that linear motion was produced succesfully. In the gap stabilization phase, linear motion resembles gap motion because of the same offset given to gap and linear displacement. Both linear motion derived from angular motion and linear encoders shows similarity, due to the gap relation in (1). The dq-axis currents for decoupling control (Fig. 13(c)) show that the decoupling controller exhibits less d-axis current during gap stabilization and q-axis currents has less fluctuations due to the inclusion of the coupled term in the control structure. Currents for independent control (Fig. 13(f)) show that larger d-axis current were used in gap stabilization and larger q-axis current fluctuations were observed. 
TABLE II

SPIRAL MOTOR EXPERIMENTAL PARAMETERS

\begin{tabular}{lll}
\hline \hline Parameters & Value & Unit \\
\hline HARDWARE PARAMETERS & & \\
\hline Spiral motor total weight & 2.3 & {$[\mathrm{~kg}]$} \\
Spiral motor full stroke & 70 & {$[\mathrm{~mm}]$} \\
Spiral motor extended length & 0.3805 & {$[\mathrm{~m}]$} \\
Spiral motor contracted length & 0.3105 & {$[\mathrm{~m}]$} \\
Spiral motor rated force (est.) & 150 & {$[\mathrm{~N}]$} \\
Inverter DC voltage (max.) & 150 & {$[\mathrm{~V}]$} \\
Inverter DC current (max.) & 6 & {$[\mathrm{~A}]$} \\
Linear encoder resolution & 1 & {$[\mu \mathrm{m}]$} \\
Rotary encoder resolution & 20000 & {$[\mathrm{pulse} / \mathrm{rev}]$} \\
Pole pairs per revolution & 2 & \\
Lead length of screw $l_{p}$ & 20 & {$[\mathrm{~mm}]$} \\
Force Constant $K_{f}$ & 20 & {$[\mathrm{Nm} / \mathrm{A}]$} \\
Torque Constant $K_{\tau}$ & 0.25 & {$[\mathrm{~N} / \mathrm{A}]$} \\
Gap Constant $K_{g}$ & 1000000 & {$[\mathrm{~N} / \mathrm{m}]$} \\
Rotor Mass $M_{s}$ & 0.7 & {$[\mathrm{~kg}]$} \\
Rotor Inertia $J_{s}$ & 0.0016 & {$\left[\mathrm{kgm}{ }^{2}\right]$} \\
\hline CONTROL PARAMETERS & & \\
\hline Control sample period & 66.7 & {$[\mu s]$} \\
Carrier frequency & 15 & {$[\mathrm{kHz}]$} \\
Proportional gain of gap, $K_{p g}$ & 1690000 & \\
Derivative gain of gap, $K_{d g}$ & 2600 & \\
Proportional gain of angle, $K_{p \tau}$ & 62500 & \\
Derivative gain of angle, $K_{d \tau}$ & 500 & \\
Proportional gain of current regulator, $K_{p i}$ & 30.303 & \\
Integral gain of current regulator, $K_{i}$ & 30303 & \\
Linear motion cut-off frequiency, $\omega_{1}$ & 796 & {$[\mathrm{~Hz}]$} \\
Angular motion cut-off frequiency, $\omega_{2}$ & 796 & {$[\mathrm{~Hz}]$} \\
Linear motion disturbance observer gain, $g_{x}$ & 111 & {$[\mathrm{~Hz}]$} \\
Angular disturbance observer gain, $g_{\theta}$ & 80 & {$[\mathrm{~Hz}]$} \\
\hline \hline
\end{tabular}

\section{CONCLUSION}

This paper explained the direct drive control of a spiral motor in monoarticular position. At first, the spiral motor structure was explained and the simplified equations of motions were shown. Then the direct drive control scheme were detailed for decoupling and independent control methods. Simulations were later performed to show that both methods were able to control gap and angular displacements with acceptable responses. Finally, experimental results validate both control methods. The advantage of independent controller is less computation required for control due to assumption of independence between d- and q-axis control of gap and angular displacement. Future works include application of the spiral motor in more complex musculoskeletal structure (i.e. biarticular position) and experimental validation of force and compliant control for the spiral motor.

\section{ACKNOWLEDGMENT}

This work was supported by KAKENHI 24246047.

\section{REFERENCES}

[1] P. Bigras, M. Lambert, C. Perron, 'Robust Force Controller for Industrial Robots: Optimal Design and Real-Time Implementation on a KUKA

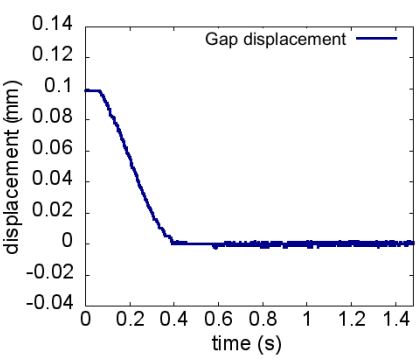

(a)

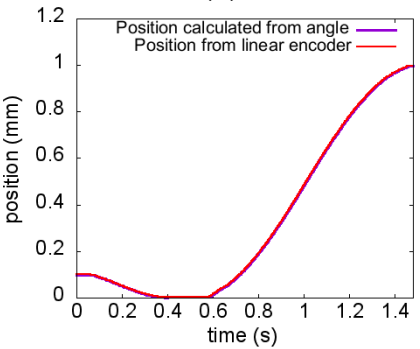

(b)

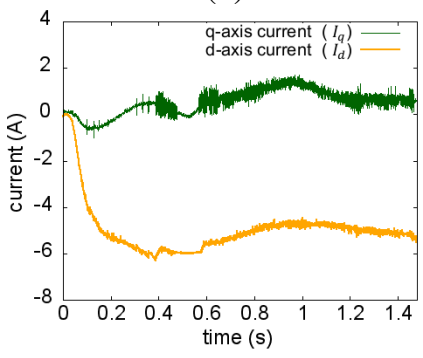

(c)

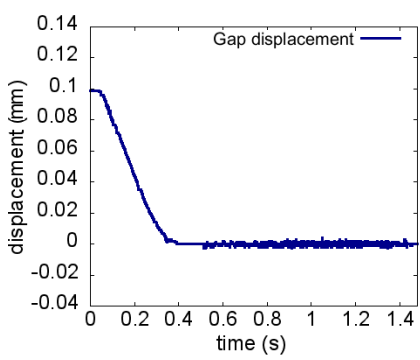

(d)

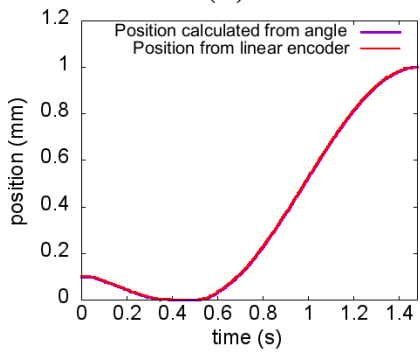

(e)

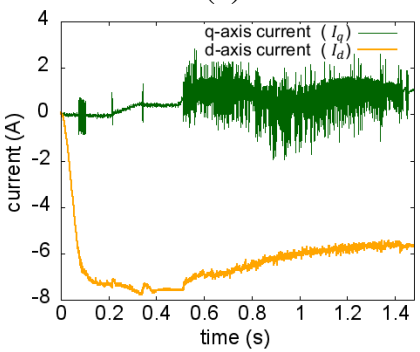

(f)
Fig. 13. Experimental results: decoupling control; (a) gap (b) position response from linear encoder and calculated value from rotary encoder (c), dqaxis currents, independent control; (d) gap (e) position response from linear encoder and calculated value from rotary encoder (f), dq-axis currents

Robot," IEEE Transactions on Control Systems Technology, Vol. 20, No. 2, pp 473-479, March 2012.

[2] I. Bonilla, E.J. Gonzalez-Galvan, C. Chavez-Olivares, M. Mendoza, A. Loredo-Flores, F. Reyes, B. Zhang, "A Vision-based, Impedance Control Strategy for Industrial Robot Manipulators," IEEE Conference on Automation Science and Engineering, pp 216-221, August 2010.

[3] M. Focchi, T. Boaventura, C. Semini, M. Frigerio, J. Buchli, D.G. Caldwell, "Torque-control based compliant actuation of a quadruped robot," IEEE International Workshop on Advanced Motion Control, pp 1-6, March 2012.

[4] M. Malchano, K. Blankespoor, A. Howardy, A.A. Rizzi, M. Raibert, "Autonomous navigation for Big-Dog," IEEE International Conference on Robotics and Automation, pp 4736 - 4741, May 2010.

[5] T.Y. Choi, J.J. Lee, "Control of Manipulator using Pneumatic Muscles for Enhanced Safety," IEEE Transactions on Industrial Electronics, Vol. 57, no. 8, pp 2815-2285, August 2010.

[6] K. Hosoda, H. Takayama, T. Takuma "Bouncing Monopod with Biomimetic Musculor-Skeleton System," International Conference on Intelligent Robots and Systems, pp 3083-3088, Sept 2008.

[7] R. Niiyama, A. Nagakubo, Y. Kuniyoshi "Mowgli: A Bipedal Jumping and Landing Robot with an Articial Musculoskeletal System," International Conference on Robotics and Automation, pp 2546-2551, April 2007.

[8] K. Abe, T. Suga, Y. Fujimoto, "Control of a Biped Robot Driven By Elastomer-based Series Elastic Actuator," 12th IEEE International Workshop on Advanced Motion Control, March 2012.

[9] N.G. Tsagarakis, Li Zhibin, J. Saglia, D.G. Caldwell, "The design of the lower body of the compliant humanoid robot cCub," IEEE International Conference on Robotics and Automation, pp 2035-2040, May 2011.

[10] M. Laffranchi, N.G. Tsagarakis, D.G. Caldwell, "Variable physical damping actuators (VPDAs): Facilitating the control and improving the 
performance of compliant actuation systems," 12th IEEE International Workshop on Advanced Motion Control, pp 1-6, March 2012.

[11] K. Kyoungchul, B. Joonbum, M. Tomizuka, "A Compact Rotary Series Elastic Actuator for Human Assistive Systems," IEEE/ASME Transactions on Mechatronics, Vol: 17, No.2, pp 288-297, April 2012.

[12] V. Salvucci, Y. Kimura, S. Oh, Y. Hori, "BiWi: Bi-articularly actuated and wire driven robot arm," IEEE International Conference on Mechatronics, pp 827-832, April 2011.

[13] T. Tsuji, "A model of antagonistic triarticular muscle mechanism for lancelet robot," IEEE International Workshop on Advanced Motion Control, pp 496-501, March 2010.

[14] V. Salvucci, Y. Kimura, S. Oh, Y. Hori, "Force Maximization of Biarticularly Actuated Manipulators Using Infinity Norm," IEEE/ASME Transactions on Mechatronics, Vol. PP, No. 99, pp 1-10, 2012.

[15] Y. Kimura, S. Oh, Y. Hori, "Novel Robot Arm with Bi-articular Driving System Using a Planetary Gear System," IEEE International Workshop on Advanced Motion Control, pp 296-301, March 2010.

[16] Y. Nakata, A. Ide, Y. Nakamura, K. Hirata, H. Ishiguro, "Hopping of a monopedal robot with a biarticular muscle driven by electromagnetic linear actuators," IEEE International Conference on Robotics and Automation, pp 3153-3160, May 2012.

[17] J.-L. Peralta, T. Ylikorpi, K. Gulzar, P. Jakubik, A. Halme, "Novel design of biped robot based on Linear Induction Motors," IEEE-RAS International Conference on Humanoid Robots, pp 34-39, December 2009.

[18] Wang Jiabin, D. Howe, Lin Zhengyu, "Design Optimization of ShortStroke Single-Phase Tubular Permanent-Magnet Motor for Refrigeration Applications," IEEE Transactions on Industrial Electronics, Vol. 57, No. 1, pp 327-334, January 2010.

[19] Lee Seungho, Kim Won-jong, "Active Suspension Control With DirectDrive Tubular Linear Brushless Permanent-Magnet Motor," IEEE Transactions on Control Systems Technology, Vol. 18, No. 4, pp 859-870, July 2010.

[20] C. Urban, R. Gunther, T. Nagel, R. Richter, R. Witt, "Development of a Bendable Permanent-Magnet Tubular Linear Motor," IEEE Transactions on Magnetics, Vol. 48, No. 8, pp 2367-2373, August 2012.

[21] F. Marignetti, S. Carbone, V. Delli Colli, C. Attaianese, "Cryogenic Characterization of Copper-Wound Linear Tubular Actuators," IEEE Transactions on Industrial Electronics, Vol. 59, No. 5, pp 2167-2177, May 2012

[22] Jibin Zou, Mei Zhao, Qian Wang, Jiming Zou, Guangkun Wu, ”Development and Analysis of Tubular Transverse Flux Machine With PermanentMagnet Excitation," IEEE Transactions on Industrial Electronics, Vol. 59, No. 5, pp 2198-2207, May 2012.

[23] Mei-Yung Chen, Tzuo-Bo Lin, Shao-Kang Hung, Li-Chen Fu, "Design and Experiment of a MacroMicro Planar Maglev Positioning System," IEEE Transactions on Industrial Electronics, Vol. 59, No. 11, pp 41284139, November 2012.

[24] Rong-Jong Wai, Jeng-Dao Lee, Kun-Lun Chuang, "Real-Time PID Control Strategy for Maglev Transportation System via Particle Swarm Optimization," IEEE Transactions on Industrial Electronics, Vol. 58, No. 2, pp 629-646, February 2011.

[25] F. Cupertino, P. Giangrande, G. Pellegrino, L. Salvatore, "End Effects in Linear Tubular Motors and Compensated Position Sensorless Control Based on Pulsating Voltage Injection," IEEE Transactions on Industrial Electronics, Vol. 58, No. 2, pp 494-502, February 2011.
[26] D. Sepasi, R. Nagamune, F. Sassani "Tracking Control of Flexible Ball Screw Drives With Runout Effect and Mass Variation," IEEE Transactions on Industrial Electronics, Vol. 59, No. 2, pp 1248-1256, February 2012.

[27] Y. Fujimoto, T. Kominami, H. Hamada "Development and Analysis of a High Thrust Force Direct-Drive Linear Actuator," IEEE Transactions on Industrial Electronics, Vol. 56, No. 5, pp 1383-1392, May 2009.

[28] I.A. Smadi, H. Omori, Y. Fujimoto, "Development, Analysis and Experimental Realization of a Direct-Drive Helical Motor," IEEE Transactions on Industrial Electronics, Vol. 59, No. 5, pp 2208-2216, May 2012.

[29] A.Z.Shukor, Y. Fujimoto, "Workspace Control of Biarticular Manipulator," The IEEE International Conference on Mechatronics, pp 415-420, April 2011.

[30] A.Z.Shukor, Y. Fujimoto, "Force Control of Musculoskeletal Manipulator," The IEEE International Workshop on Advanced Motion Control, pp 415-420, March 2012.

[31] A.Z. Shukor, Y. Fujimoto, "Load Disturbance and Environment Effect on Biarticular Manipulator driven by Spiral Motors," The IEEE International Conference on Mechatronics, ID-002593, March 2013.

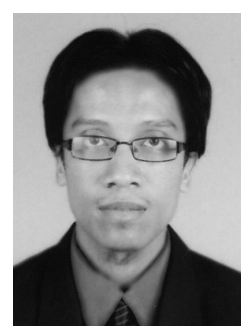

Ahmad Zaki Shukor (M'12) received the B.E in electrical engineerng from Universiti Teknologi Malaysia in 2002 and M.E. in electrical engineering from University of South Australia in 2004. He obtained a $\mathrm{PhD}$ degree in electrical and computer engineering in Yokohama National University, Japan, in 2013

Since 2002, he has been attached with the Faculty of Electrical Engineering, Universiti Teknikal Malaysia Melaka, where he is currently a Senior Lecturer. His research interests include actuators, robotics, and motion control

$\mathrm{He}$ is a graduate member of the Board of Engineers Malaysia.

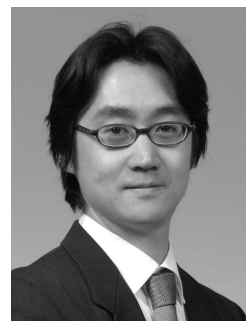

Yasutaka Fujimoto (S'93-M'98-SM'12) received the B.E., M.E., and Ph.D. degrees in electrical and computer engineering from Yokohama National University, Yokohama, Japan, in 1993, 1995, and 1998, respectively.

In 1998, he joined the Department of Electrical Engineering, Keio University, Yokohama, Japan, as a Research Associate. Since 1999, he has been with the Department of Electrical and Computer Engineering, Yokohama National University, where he is currently a Professor. His research interests include actuators, robotics, manufacturing automation, and motion control.

Dr. Fujimoto is a chair of the IEEE Industrial Electronics Society Technical Committee on Sensors and Actuators (2012-2013), Institute of Electrical Engineers of Japan, and Robotics Society of Japan. 